\title{
The Evolutionary Evidence for Most Be Stars Being Paired with Hot White Dwarfs
}

\author{
Natalya V. Raguzova \\ Sternberg Astronomical Institute, Moscow University, Moscow, 119899 \\ Russia
}

\begin{abstract}
Using the "Scenario Machine" we study the number and physical properties of binary Be stars with white dwarfs taking into account cooling of the compact object and discuss the formation of such systems. According to our calculations the number of binary systems containing a Be star paired with a white dwarf in our galaxy is very large $-46 \%$ of all Be stars formed as a result of binary evolution must have a white dwarf as companion. These white dwarfs should be very hot with a surface temperature lying in the range from 30,000 to $60,000 \mathrm{~K}$.
\end{abstract}

\section{Introduction}

$\mathrm{Be}$ stars in $\mathrm{Be} / \mathrm{X}$-ray binaries turn out to cover a very limited range in spectral type, namely between $\mathrm{O} 9$ and $\mathrm{B} 2$. This is due to the fact that only the more massive systems can produce a neutron star. However, evolutionary calculations show that the less massive systems will produce a rapidly rotating $\mathrm{Be}$ star, which may be of later spectral type than B2, and a white dwarf. In this paper we make a population computation of the abundance of Be/WD binaries, calculate their expected observational characteristics and discuss their influence on observational appearances of white dwarfs in binary system with a Be star. We also discuss a possible way of detection of a white dwarf in such a system.

\section{The problem of white dwarf cooling}

The dominant source of luminosity of a white dwarf is the consumption of thermal energy of the ions stored in the stellar interior. The theory of cooling of single white dwarfs (Kaplan 1950; Mestel 1952) predicts the connection between the luminosity of the white dwarf and its age, which is confirmed well by observational data. Without going into detail of the theory we give simply the basic formula obtained by us for the dependence of the luminosity of the white dwarf on time, $L(t)$ :

$L(t)=1.82 \times 10^{30} \frac{\mu}{\mu_{Z}^{2}} \frac{M / M_{\odot}}{x_{Z}\left(1+x_{H}\right)}\left(\frac{\tau}{1.72 \times 10^{10}} \frac{A}{x_{Z}\left(1+x_{H}\right)} \frac{\mu}{\mu_{Z}^{2}}+T_{7,0}^{-2.5}\right)^{-7 / 5}$

where $\mu$ is the mean atomic weight, $\mu_{Z}$ is the number of nucleons per electron, $M$ is the white dwarf mass, $x_{Z}$ is the fraction of heavy elements (all elements other than $\mathrm{H}$ and $\mathrm{He}$ ), $x_{H}$ is the fraction of $\mathrm{H}, \tau$ is the cooling time of a white 

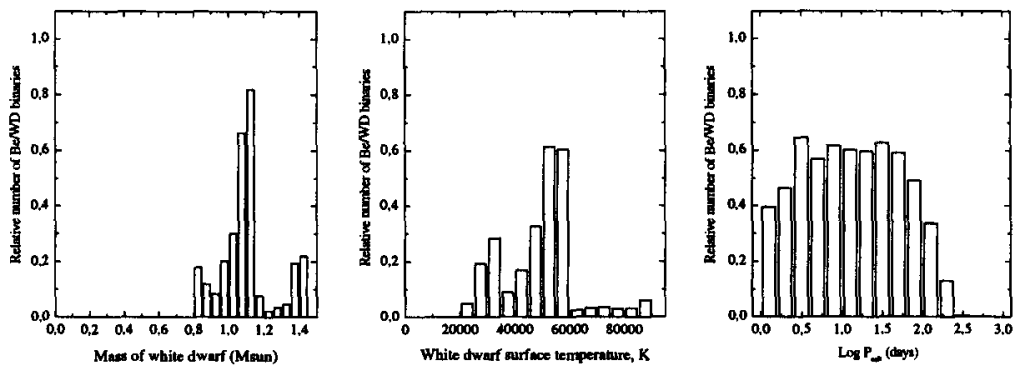

Figure 1. The expected distribution of the number of Be/WD binaries over different physical properties

dwarf with core temperature between $T_{0}$ and $T$ (in years), $T_{7}=T \times 10^{-7}$, $A$ is the atomic weight. It is commonly taken that in the envelope of white dwarfs $x_{Z}=0.1, x_{H}=0$, then, $\mu_{Z}=2, \mu=1.38, \mu /\left[\mu_{Z}^{2} x_{Z}\left(1+x_{H}\right)\right]=3.45$. Evidently if we know the initial temperature of the core then we can calculate the temperature at the surface of the white dwarf, $T_{\text {eff }}$, for any moment of time.

\section{The population synthesis of binary Be stars paired with white dwarfs and their physical properties}

The calculations have been made using the "Scenario Machine" (a numerical code that models the evolution of large ensembles of binary systems; Lipunov et al. 1996).

The existence of white dwarf companions of Be stars is of significant importance from an evolutionary standpoint since they must have evolved from massive progenitors, perhaps close to the maximum mass for white dwarf progenitor stars, and they are likely themselves to be much more massive than the mean for white dwarfs in general $\left(0.57 \mathrm{M}_{\odot}\right.$, Finley, Koester \& Basri 1997). Really, the presence of a component of spectral class B provides a good reason to think that the white dwarf progenitor star was massive enough to evolve faster and leave the main sequence and, then, collapse into a white dwarf. The spectral type of the normal star in these binaries gives a lower limit for the mass of the white dwarf progenitor. It is evident that the maximum of the distribution of the number of $\mathrm{Be} / \mathrm{WD}$ binaries over masses with a degenerate companion in distinction to similar distributions for single white dwarfs and their analogs in low-mass binary systems must shift to larger values of the mass. The expected distribution of the number of Be/WD binaries over masses of the compact object is shown in Fig. 1. The peak of the distribution is at the value $1.1 \mathrm{M}_{\odot}$ and hence the previous assumption is true.

The expected distribution of the number of $\mathrm{Be} / \mathrm{WD}$ binaries over the surface temperatures of white dwarfs is shown in Fig. 1. The initial temperature of the core for the calculations is equal to $5 \times 10^{7} \mathrm{~K}$ by recognizing that the maximum value of the surface temperature of a white dwarf obtained from observations 


\begin{tabular}{|c|c|c|c|c|c|c|}
\hline & & M2 & A & $\mathbf{T}$ & $P_{\text {ort }}$ \\
\hline \multicolumn{2}{|c|}{ Solar masses } & \multicolumn{2}{|c|}{ Solar masses } & Solar radii & Million years & Days \\
\hline 9.10 & MS & MS & 8.00 & 50.16 & 0.00 & 10.0 \\
\hline 8.90 & RLO (fast) & MS & 7.88 & 14.19 & 21.27 & 1.5 \\
\hline 8.27 & RLO (slow) & MS & 8.27 & 14.25 & 21.28 & 1.5 \\
\hline 1.07 & Hot core 6 & $\mathrm{Be}$ & 12.85 & 252.40 & 26.60 & 125.1 \\
\hline 1.07 & WD & $\mathrm{Be}$ & 12.84 & 252.60 & 26.71 & 125.3 \\
\hline
\end{tabular}

Figure 2. A possible evolutionary track leading to Be/WD binary formation

is equal to $70,000-80,000 \mathrm{~K}$. Based on our calculations we conclude that most of the white dwarfs paired with Be stars must be very hot with surface temperatures lying in the range from 30,000 to $60,000 \mathrm{~K}$. This can be explained from the fact that white dwarfs in such systems have no time to cool down in the time of the Be star evolution.

A possible evolutionary track leading to Be/WD binary formation is shown in Fig. 2. We use the following notation for the particular evolutionary stages of the components: MS = a main sequence star inside its Roche lobe (RL); a MSstar accreting matter during the first mass transfer is considered to be a rapidly rotating Be-star if its mass is more than $10 \mathrm{M}_{\odot} ; \mathrm{RLO}=\mathrm{a}$ MS or post-MS star filling its Roche lobe and transferring matter onto the companion; $\mathrm{RLO}$ (fast) = Roche lobe overflow on a thermal timescale; $\mathrm{RLO}($ slow) = slow (evolutionary driven) phase of mass transfer; Hot core $=$ a naked star core left after mass exchange - after its cooling the white dwarf forms; WD = white dwarf.

The distribution of the number of $\mathrm{Be}$ stars paired with white dwarfs over orbital periods is shown in Fig. 1. It is seen that such systems have no orbital periods more than one year, that is, all these binaries are close enough.

\section{Discussion}

According to our calculations the number of binary systems containing a Be star paired with a white dwarf is very large $-46 \%$ of all Be stars formed as a result of binary evolution must have a white dwarf as a companion (see Fig. 3). However, so far no such system have been identified. Our calculations show that all white dwarfs in systems with Be stars must be very hot (see Fig. 1). Could the presence of a white dwarf be established by the detection of its EUV continuum shortward of the Be star's continuum turnover at $1000 \AA$ ?

Apart from the fact that a white dwarf paired with a Be star must be very hot, accretion of matter can occur onto the white dwarf. It is known that 
the X-ray observations of cataclysmic variables which contain a white dwarf accreting matter from a companion, have shown that the emitted spectrum has a blackbody component with a temperature corresponding to about $40 \mathrm{eV}$ and a higher energy component with $k T$ about a few $\mathrm{keV}$. The bulk of the emission energy is in the blackbody component with the higher energy component having an energy less by factor 30 to 300 .

From our calculations it follows that the entire orbit of white dwarf is embedded in the dense circumstellar envelope of the primary star. The extremeultraviolet and soft X-ray photons are absorbed by the gas in the envelope of the Be star in which the white dwarf is embedded.

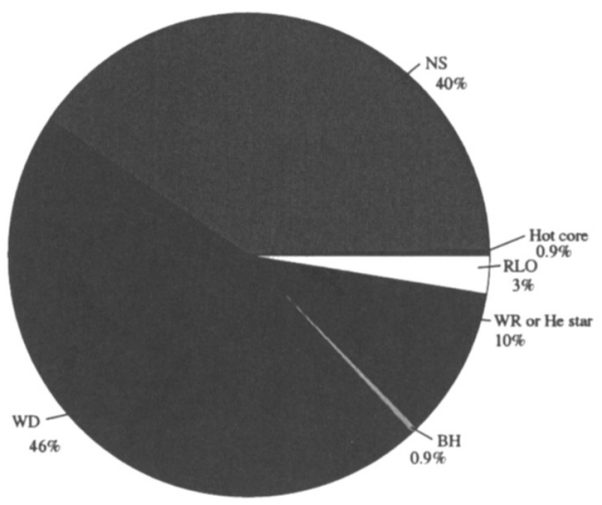

Figure 3. The fractions of binary systems containing Be stars with different companions

Thus, the suggestion about the detection of white dwarfs paired with Be stars by the detection of its extremely UV continuum shortward of the Be star's continuum turnover at $1000 \AA$, where the primary star ceases to give the dominant contribution to the emission, is of little use in practice. All extremely UV and soft X-ray photons of white dwarfs are absorbed by the Be star envelope. However, the suggestion above is applicable to a binary Be/WD system during a period without a $\mathrm{Be}$ star disc-like envelope.

Acknowledgments. This work is partially supported by the RFBR grant 98-02-16801, and by the "Universitety Rossii" grant 5559.

\section{References}

Finley, D.S., Koester, D., Basri, G. 1997, ApJ 488, 375

Kaplan, S.A. 1950, AZh 27, 31

Lipunov, V.M., Postnov, K.A., Prokhorov, M.E. 1996, Astrophys. and Space Phys. Reviews, 17, 1

Mestel, L. 1952, MNRAS 112, 583 